\title{
International Journal of Statistics and Applied Mathematics
}

ISSN: $2456-1452$

Maths 2021; 6(5): 54-58

(C) 2021 Stats \& Maths

www.mathsjournal.com

Received: 19-07-2021

Accepted: 21-08-2021

Arup Kumar Saha

Department of Education in

Science and Mathematics

Regional Institute of Education

(NCERT), Bhubaneswar,

Odisha, India

\section{Manoj Kumar Hota}

Department of Mathematics,

Nayagarh Autonomous College,

Nayagarh, Odisha, India

Prasanta Kumar Mohanty

Department of Mathematics,

School of Applied Sciences

KIIT Deemed to be University,

Bhubaneswar, Odisha, India

Corresponding Author:

Arup Kumar Saha

Department of Education in

Science and Mathematic

Regional Institute of Education

(NCERT), Bhubaneswar,

Odisha, India

\section{Approximate evaluation of complex hyper singular integrals}

\section{Arup Kumar Saha, Manoj Kumar Hota and Prasanta Kumar Mohanty}

DOI: https://doi.org/10.22271/maths.2021.v6.i5a.726

\section{Abstract}

In this paper we develop a method for approximate evaluation of complex hyper singular integrals in the complex plane. The schemes are numerically validated using a set of conventional test integrals. A number of examples is provided to illustrate the the efficiency of the method develop here.

Keywords: analytic function, cauchy principal value, Hardamad finite part integral, Teylor's coefficients

\section{Introduction}

Integrals of the type

$I\left(f, z_{0}\right)=H \int_{L} \frac{f(z)}{\left(z-z_{0}\right)^{\alpha}} d z ; \alpha \in \mathbb{N}-\{1\}$

are frequently appeared in contour integration, where $f(z)$ is infinitely differentiable function in $\Omega=\left\{z \in \mathbb{C}:\left|z-z_{0}\right|<\rho=r|h|, r>1\right\}$;

of the complex plane $\mathbb{C}$ and $L$ joining the points $z_{0}-h$ to $z_{0}+h$ lying in the disc $\Omega$.

It is seen that rules (Ref. ${ }^{[4,7,8,9,12,15]}$ ) meant for the numerical integration of the integral $I=$ $\int \frac{f(z)}{z-z_{0}} d z$

lead to uncontrolled instability when those are applied for the approximation of the integral given in equation (1). This is due to the presence of singular point $z_{0}$ of order $\alpha>1$ on the path of integration $L$. The integral defined in equation (1) is called as hyper singular integral in complex plane. The study of its real counter part has been going on for a long time and has been documented in a number of publications (Ref. ${ }^{[6,13,[?], 17-22]}$ ).

$J^{*}=H \int_{a}^{b} \frac{f(x)}{(x-c)^{2}} d x ; a<c<b$.

However, a very few rules in the course of numerical integration have devised for the former. Therefore, in this study we have proposed a numerical scheme for the numerical computation of the integral given in equation (1).

2. Description of the scheme for numerical evaluation of complex hyper singular integral To establish the scheme for the numerical computation of the hyper singular integral

$I\left(f, z_{0}\right)=H \int_{L} \frac{f(z)}{\left(z-z_{0}\right)^{\alpha}} d z ; \alpha \in \mathbb{N}-\{1\} ;$

we assume here that the function $f(z)$ is analytic and infinitely differentiable on the disc

$\Omega=\left\{z \in \mathbb{C}:\left|z-z_{0}\right|<\rho=r|h|, r>1\right\}$ 
With this assumption expanding $f(z)$ about the point $z=z_{0}$ we obtain

$f(z)=\sum_{k=0}^{\infty} c_{k}\left(z-z_{0}\right)^{k}$

where $c_{k}=\frac{f^{(k)}\left(z_{0}\right)}{k !}$ is the Taylor's coefficient.

Since

$$
\begin{aligned}
f(z) & =\sum_{k=0}^{\infty} c_{k}\left(z-z_{0}\right)^{k} \\
& =\sum_{k=0}^{\alpha-2} c_{k}\left(z-z_{0}\right)^{k}+\sum_{k=\alpha-1}^{\infty} c_{k}\left(z-z_{0}\right)^{k}
\end{aligned}
$$

Thus,

$$
\begin{aligned}
& f(z)-\sum_{k=0}^{\alpha-2} c_{k}\left(z-z_{0}\right)^{k}=\sum_{k=\alpha-1}^{\infty} c_{k}\left(z-z_{0}\right)^{k} \\
& \frac{f(z)-\sum_{k=0}^{\alpha-2} c_{k}\left(z-z_{0}\right)^{k}}{\left(z-z_{0}\right)^{\alpha-1}}=\sum_{k=\alpha-1}^{\infty} c_{k}\left(z-z_{0}\right)^{k-\alpha+1}
\end{aligned}
$$

$=\sum_{j=0}^{\infty} c_{j+\alpha-1}\left(z-z_{0}\right)^{j}=g(z)$

for $j=k-\alpha+1$.

As a result $g(z)$ is analytic in $\Omega$.

Therefore,

$$
\begin{aligned}
I\left(f, z_{0}\right) & =H \int_{L} \frac{f(z)}{\left(z-z_{0}\right)^{\alpha}} d z \\
& =P \int_{L} \frac{g(z)}{z-z_{0}} d z+\sum_{k=0}^{\alpha-2} \int_{L} \frac{c_{k}}{\left(z-z_{0}\right)^{\alpha-k}} \\
& =I_{g}+\sum_{k=0}^{\alpha-2} I_{\alpha k} ;
\end{aligned}
$$

where

$I_{g}=P \int_{L} \frac{g(z)}{z-z_{0}} d z$

and

$I_{\alpha k}=\int_{L} \frac{c_{k}}{\left(z-z_{0}\right)^{\alpha-k}}$

Since the function $g(z)$ is analytic in the domain $\Omega$ thus, the first integral appears in the right side of the equation (3) is a Cauchy type singular integral with singularity of order one. Hence, any quadrature rule meant for the numerical integration of the complex CPV integral may be applied for the numerical approximation of the integral given in equation (4).

However, it is well known that the integral

$I_{\alpha k}=\int_{L} \frac{c_{k}}{\left(z-z_{0}\right)^{\alpha-k}}$

is analytically a diverging integral and diverges for $\alpha-k>1$. Moreover it is a hyper singular integral and its finite part (Hadmard finite part) can be evaluated by transforming the integral onto the real axis with the help of the transformation

$z=z_{0}+h t ;-1 \leq t \leq 1$

(Ref. Davis and Rabinowitz, ${ }^{[10]}$, Kai Diethelm ${ }^{[11]}$, Ang, W.T ${ }^{[1]}$, Ang, W.T and Clements, D.L ${ }^{[2]}$, Hasegawa.T ${ }^{[14]}$ and A.R. Krommer and C.W. Ueberhuber ${ }^{[16]}$ )

Now, by using this transformation the integral given in equation (5) is reduced into 


$$
\begin{aligned}
I_{\alpha k} & =\int_{z_{0}-h}^{z_{0}+h} \frac{c_{k}}{\left(z-z_{0}\right)^{\alpha-k}} d z \\
& =\frac{c_{k}}{h^{\alpha-k-1}} \int_{-1}^{1} \frac{d t}{t^{\alpha-k}}
\end{aligned}
$$

$=\left\{\begin{array}{lll}\frac{2 c_{k}}{h^{\alpha-k-1}} \int_{0}^{1} \frac{d t}{t^{\alpha-k}} & , \text { for } & \alpha-k \text { is even } \\ 0 & , \text { for } & \alpha-k \text { is odd } .\end{array}\right.$

The integral appears for $\alpha-k$ as even i.e.

$\int_{0}^{1} \frac{d t}{t^{\alpha-k}}$

is a hyper singular integral in real axis. To evaluate this integral, we consider the convergent integral

$\int_{\epsilon}^{1} \frac{d t}{t^{\alpha-k}}$

The value of the convergent integral can be simply obtained as

$\frac{1-\epsilon^{k-\alpha+1}}{k-\alpha+1}=\frac{1}{k-\alpha+1}-\frac{1}{k-\alpha+1} \frac{1}{\epsilon^{\alpha-k-1}}$

Now letting $\epsilon \rightarrow 0$; of course the limit does not exist and so Hadamard suggested to simply ignore the unbounded contribution of $\lim _{\epsilon \rightarrow 0} \frac{1}{\epsilon}$ (Ref. Kai Diethelm ${ }^{[11]}$, pp.233) and to assign the value of remaining finite expression.

As a result, the finite part value of our original integral

$I_{\alpha k}=\left\{\begin{array}{lll}\frac{2 c_{k}}{h^{\alpha-k-1}(k-\alpha+1)} & , \text { for } & \alpha-k \text { is even } \\ 0 & , \text { for } & \alpha-k \text { is odd }\end{array}\right.$

It is noteworthy to mention here that "as far as equality is concerned, the common rules for ordinary integrals are also valid for finite part integrals, but rules concerning inequalities are not applicable".(Ref. pp.13, Ang, W.T ${ }^{[1]}$, Ang, W.T and Clements, D.L ${ }^{[2]}$, Davis and Rabinowitz, ${ }^{[10]}$ )

Therefore, the integral

$I\left(f, z_{o}\right) \approx R_{g}+\sum_{k=0}^{\alpha-2} \frac{2 c_{k}}{h^{\alpha-k-1}(k-\alpha+1)} ;$

where $R_{g}$ is the quadrature rule (constructed in chapter-ch:comp) meant for the numerical integration of the complex CPV integral $I_{g}$.

To verify the accuracy of the proposed scheme numerically, we have applied the scheme over some standard test integrals already considered by different researchers. The results of their numerical approximations are given in Table- 1 and Table- 2 in the section of Numerical experiments.

\section{Numerical experiments}

\subsection{Evaluation of complex strongly singular integrals}

In this subsection we have considered the hyper singular integrals

$\Gamma_{1}=H \int_{-i}^{i} \frac{e^{z}}{z^{2}} d z ; \quad \Gamma_{2}=H \int_{-i}^{i} \frac{e^{z}}{z^{3}} d z$

$\Gamma_{3}=H \int_{-i}^{i} \frac{e^{z}}{z^{4}} d z ; \quad \Gamma_{4}=H \int_{-i}^{i} \frac{e^{z}}{z^{5}} d z ;$

Since the scheme (as constructed in Subsection-2) has established by incorporating the rules meant for the numerical integration of complex CPV integrals therefore, here we have employed the scheme with the help of the rules for CPV integrals in order of their increasing algebraic degree of precession for the approximate evaluation of these above integrals. The approximate value of the integrals with their absolute errors are reflected in Table-1 and Table-2.

The basic purpose of numerically evaluating an integral (either singular or nonsingular) by a sequence of rules of increasing precession is to establish the fact that the final approximation corresponding to the rule of highest precession is possibly the best available approximation at hand. Because, from the trend of approximate values resulting from numerical integration of any integral (either definite or CPV integral) by a sequence of rules from lowest precession to highest precession will suggest to 
accept or not to accept the results of numerical integration by the highest precession as the value (Approximate value) of an integral; which one cannot be assured of by applying a single quadrature rule.

Keeping this in mind, we have evaluated numerically the above integrals by a sequence of rules instead of a single quadrature rule. The approximate values corresponding to the rule of highest precession have been taken into account for calculation of final approximate value of integrals.

Table 1: Numerical evaluation of complex hyper singular integrals

\begin{tabular}{|c|c|c|c|c|}
\hline Rules & Approximate Value of $\boldsymbol{\Gamma}_{\mathbf{1}}$ & Absolute Error & Approximate Value of $\boldsymbol{\Gamma}_{\mathbf{2}}$ & Absolute Error \\
\hline$Q_{1}(f)$ & $2.972769271793576 i$ & $1.5 \times 10^{-6}$ & $2.327856195900890 i$ & $1.6 \times 10^{-7}$ \\
\hline$Q_{2}(f)$ & $2.972770934810439 i$ & $1.8 \times 10^{-7}$ & $2.327856381435858 i$ & $2.0 \times 10^{-8}$ \\
\hline$Q_{3}(f)$ & $2.972770804264940 i$ & $5.2 \times 10^{-8}$ & $2.327856366882020 i$ & $5.8 \times 10^{-9}$ \\
\hline$R_{1}(f)$ & $2.972770688067246 i$ & $6.4 \times 10^{-8}$ & $2.327856355188614 i$ & $5.8 \times 10^{-9}$ \\
\hline$R_{2}(f)$ & $2.972770776296036 i$ & $2.4 \times 10^{-8}$ & $2.327856363211525 i$ & $2.2 \times 10^{-9}$ \\
\hline$R_{3}(f)$ & $2.972770763520918 i$ & $1.1 \times 10^{-8}$ & $2.327856362049843 i$ & $1.0 \times 10^{-9}$ \\
\hline$Q_{32}(f)$ & $2.972770749298414 i$ & $3.2 \times 10^{-9}$ & $2.327856360754087 i$ & $2.9 \times 10^{-10}$ \\
\hline$Q_{13}(f)$ & $2.972770749533820 i$ & $2.9 \times 10^{-9}$ & $2.327856360775551 i$ & $2.7 \times 10^{-10}$ \\
\hline$Q_{12}(f)$ & $2.972770750030788 i$ & $2.4 \times 10^{-9}$ & $2.327856360820862 i$ & $2.2 \times 10^{-10}$ \\
\hline$R_{12}(f)$ & $2.972770752342519 i$ & $1.3 \times 10^{-10}$ & $2.327856361033359 i$ & $9.9 \times 10^{-12}$ \\
\hline$R_{13}(f)$ & $2.972770752342596 i$ & $1.3 \times 10^{-10}$ & $2.327856361033365 i$ & $9.8 \times 10^{-12}$ \\
\hline$R_{23}(f)$ & $2.972770752342690 i$ & $1.3 \times 10^{-10}$ & $2.327856361033371 i$ & $9.8 \times 10^{-12}$ \\
\hline$T_{1}(f)$ & $2.972770752411771 i$ & $5.9 \times 10^{-11}$ & $2.327856361038685 i$ & $4.5 \times 10^{-12}$ \\
\hline$T_{2}(f)$ & $2.972770752415014 i$ & $5.6 \times 10^{-11}$ & $2.327856361038938 i$ & $4.3 \times 10^{-12}$ \\
\hline$T_{3}(f)$ & $2.972770752421825 i$ & $4.9 \times 10^{-11}$ & $2.327856361039460 i$ & $3.8 \times 10^{-12}$ \\
\hline
\end{tabular}

Table 2: Numerical evaluation of complex hyper singular integrals

\begin{tabular}{|c|c|c|c|c|}
\hline Rules & Approximate Value of $\boldsymbol{\Gamma}_{3}$ & Absolute Error & Approximate Value of $\boldsymbol{\Gamma}_{4}$ & Absolute Error \\
\hline$Q_{1}(f)$ & $0.415750566541269 i$ & $1.7 \times 10^{-8}$ & $-0.316797848137200 i$ & $1.5 \times 10^{-9}$ \\
\hline$Q_{2}(f)$ & $0.415750585151556 i$ & $2.0 \times 10^{-9}$ & $-0.316797846441370 i$ & $1.9 \times 10^{-10}$ \\
\hline$Q_{3}(f)$ & $0.415750583692510 i$ & $5.9 \times 10^{-10}$ & $-0.316797846574279 i$ & $5.4 \times 10^{-11}$ \\
\hline$R_{1}(f)$ & $0.415750582614445 i$ & $4.9 \times 10^{-10}$ & $-0.316797846665897 i$ & $3.7 \times 10^{-11}$ \\
\hline$R_{2}(f)$ & $0.415750583283161 i$ & $1.8 \times 10^{-10}$ & $-0.316797846614448 i$ & $1.4 \times 10^{-11}$ \\
\hline$R_{3}(f)$ & $0.415750583186333 i$ & $8.4 \times 10^{-11}$ & $-0.316797846621900 i$ & $6.5 \times 10^{-12}$ \\
\hline$Q_{32}(f)$ & $0.415750583078175 i$ & $2.4 \times 10^{-11}$ & $-0.316797846630241 i$ & $1.9 \times 10^{-12}$ \\
\hline$Q_{13}(f)$ & $0.415750583079966 i$ & $2.2 \times 10^{-11}$ & $-0.316797846630098 i$ & $1.7 \times 10^{-12}$ \\
\hline$Q_{12}(f)$ & $0.415750583083746 i$ & $1.9 \times 10^{-11}$ & $-0.316797846629796 i$ & $1.4 \times 10^{-12}$ \\
\hline$R_{12}(f)$ & $0.415750583101609 i$ & $7.0 \times 10^{-13}$ & $-0.316797846628416 i$ & $4.6 \times 10^{-14}$ \\
\hline$R_{13}(f)$ & $0.415750583101609 i$ & $7.0 \times 10^{-13}$ & $-0.316797846628418 i$ & $4.8 \times 10^{-14}$ \\
\hline$R_{23}(f)$ & $0.415750583101609 i$ & $7.0 \times 10^{-13}$ & $-0.316797846628420 i$ & $5.0 \times 10^{-14}$ \\
\hline$T_{1}(f)$ & $0.415750583101969 i$ & $3.4 \times 10^{-13}$ & $-0.316797846628412 i$ & $4.1 \times 10^{-14}$ \\
\hline$T_{2}(f)$ & $0.415750583102004 i$ & $3.1 \times 10^{-13}$ & $-0.316797846628342 i$ & $2.7 \times 10^{-14}$ \\
\hline$T_{3}(f)$ & $0.415750583101921 i$ & $3.9 \times 10^{-13}$ & $-0.316797846628402 i$ & $3.1 \times 10^{-14}$ \\
\hline
\end{tabular}

\section{References}

1. Ang WT. A Beginner's Course in Boundary Element Methods, Universal Publishers, 2007

2. Ang WT, Clements DL. Hypersingular integral equations for a thermoelastic problem of a multiple planar cracks in an anisotropic medium, Engineering Analysis with Boundary Elements 1999;23:713-720

3. Atkinson, Kendall. An Introduction to Numerical Analysis, John Wiley and sons, 2nd edition, 1978.

4. Bej M, Hota MK, Das RN. Numerical evaluation of real Cauchy principal value of integral in adaptive environment, Research Journal of Environmental Sciences, 2012;6:186-195

5. Brikhoof G, Young D. Numerical quadrature of analytic and harmonic functions, J. Math. Phys. sci 1950;29:217-221

6. Criscuolo G. A new algorithm for Cauchy principal value and Hadamard finite part integrals, Journal of Computational and Applied Mathematics 1997;78:255-275.

7. Das RN, Hota MK. A derivative free quadrature rule for numerical approx. of complex Cauchy principal value integrals, Applied Mathematical Sciences 2012;6(111):5533-5540.

8. Das RN, Hota MK, Bej M. Some derivative-free quadrature rules for numerical approximations of Cauchy principal value of integrals, ISRN Computational Mathematics

9. Elliot, Paget. Gauss type quadrature rules for Cauchy principal value integrals, Mathematics of Computation 1979;33:301309.

10. Davis PJ, Rabinowitz P. Methods of Numerical Integration, Second Edition, Academic Press, INC., New York, 1984.

11. Two general methods for the numerical approximation of multidimensional Cauchy principal value integrals PDF Kai Diethelm Anziam J. 2000/01; 42:E1- E26, MathSciNet.

12. Chawla MM, Jayrajan N. Quadrature formulas for Cauchy principal value integrals, Computing 1975;15:347-355. 
13. Groetsch CW. Regularized product integration for Hadamard finite part integrals, Mathematics and Computation 1995;30:3-6

14. Hasegawa T. Numerical integration of functions with poles near the interval of integration, Journal of Computational and Applied Mathematics 1997;87(2):339-357

15. He G, Xiang S. An improved algorithm for the evaluation of Cauchy principal value integrals of oscillatory functions and its application Numer 1975;15347-355.

16. Krommer AR, Ueberhuber CW. Computational Integration, Society for Industrial and Applied Mathematics

17. Ramm AG, Vander Sluis A. Calculating singular integrals as an ill-posed problem, Numerische Mathematik 1990;57:139-145

18. Sun W, Wu J. Newton-Cotes formulae for the numerical evaluation of certain hypersingular integral, Computing 2005;75:297-309.

19. Sun W, Wu J. Interpolatory quadrature rules for Hadamard finite-part integrals and their superconvergence, IMA. J. Numer. Anal 2009;28:580-597.

20. Tong M, Chew W. A novel approach for evaluating hypersingular and strongly singular surface integrals in electromagnetics, IEEE Trans. Antennas and Propagation 2010;58:3593-3601 .

21. Wang H, Xiang S. On the evaluation of Cauchy principal value integrals of oscillatory functions, Journal of Computational and Applied Mathematics 2010;234:95-100.

22. Zhang X, Gunzhurger M, Ju L. Nodal-type collocation methods for hypersingular integral equations and nonlocal diffusion problems, Comput. Meth. Appl. Mech. Eng 2016;299:401-420 . 